\title{
SCIENTIFIC REPORTS

\section{Optogenetic stimulation of the VTA modulates a frequency-specific gain of thalamocortical inputs in infragranular layers of the auditory cortex}

\author{
Michael G. K. Brunk ${ }^{1 *}$, Katrina E. Deane ${ }^{1}$, Martin Kisse ${ }^{1}$, Matthias Deliano ${ }^{1}$, Silvia Vieweg ${ }^{1}$, \\ Frank W. Ohl ${ }^{1,2,3}$, Michael T. Lippert ${ }^{1,2} \&$ Max F. K. Happel ${ }^{1,3^{*}}$
}

Reward associations during auditory learning induce cortical plasticity in the primary auditory cortex. A prominent source of such influence is the ventral tegmental area (VTA), which conveys a dopaminergic teaching signal to the primary auditory cortex. Yet, it is unknown, how the VTA influences cortical frequency processing and spectral integration. Therefore, we investigated the temporal effects of direct optogenetic stimulation of the VTA onto spectral integration in the auditory cortex on a synaptic circuit level by current-source-density analysis in anesthetized Mongolian gerbils. While auditory lemniscal input predominantly terminates in the granular input layers III/IV, we found that VTA-mediated modulation of spectral processing is relayed by a different circuit, namely enhanced thalamic inputs to the infragranular layers $\mathrm{Vb} / \mathrm{Vla}$. Activation of this circuit yields a frequency-specific gain amplification of local sensory input and enhances corticocortical information transfer, especially in supragranular layers $\mathrm{I} / \mathrm{II}$. This effects persisted over more than 30 minutes after VTA stimulation. Altogether, we demonstrate that the VTA exhibits a long-lasting influence on sensory cortical processing via infragranular layers transcending the signaling of a mere reward-prediction error. We thereby demonstrate a cellular and circuit substrate for the influence of reinforcement-evaluating brain systems on sensory processing in the auditory cortex.

The sensory cortex receives both bottom-up input relaying stimulus information from the sensory epithelia and top-down input from, for example, reinforcement-evaluating brain structures ${ }^{1}$. Among the latter, the ventral tegmental area (VTA) is a key structure associated with the coding of reward, reward prediction, and reward prediction error ${ }^{2}$. Especially in the framework of reward prediction error coding, projections of dopamine (DA) neurons in the VTA to the striatum and prefrontal cortex have been investigated in great detail ${ }^{3}$. Previous studies in awake and anaesthetized rats utilizing electrical stimulation of the VTA paired with a specific target frequency over days have shown to successfully re-organize the frequency mapping of the auditory cortex (ACx) in favor of the paired tone frequency ${ }^{4,5}$. Yet, the anatomical projections from the VTA towards sensory cortices ${ }^{6-8}$, and consequently their immediate impact on the layer-specific cortical circuit processing, have remained rather elusive.

Dopamine released in the sensory cortex may complement bottom-up stimulus processing with a behaviorally relevant representation of stimulus value and salience to support adaptive behavior ${ }^{25,9,10}$. Consistently, for the case of the ACx, intracortical dopamine levels ${ }^{11}$ and their experimental manipulation ${ }^{12,13}$ were shown to affect behavioral measures in auditory learning scenarios. Thus, dopamine appears to be involved in several general behaviorally relevant functions, including auditory perceptual decision making, prediction, and learning, which are increasingly realized to be supported by the $\mathrm{ACx}^{14}$. In agreement, we have previously shown that the pharmacological stimulation of D1/D5 receptors, which are dominantly expressed in supragranular (I/II) and

${ }^{1}$ Department of Systems Physiology of Learning, Leibniz Institute for Neurobiology, 39118, Magdeburg, Germany. ${ }^{2}$ Center for Behavioral Brain Sciences (CBBS), 39106, Magdeburg, Germany. Institute for Biology, Otto-von-GuerickeUniversity, 39120, Magdeburg, Germany. *email: mbrunk@lin-magdeburg.de; mhappel@lin-magdeburg.de 
infragranular (V/VI) layers ${ }^{13,15,16}$, influences sensory processing at the level of both local and wide-spread circuits in auditory cortex ${ }^{17}$.

In order to determine the contribution of VTA projections to these cortical effects of dopamine, we optogenetically stimulated the projection neurons of the VTA in adult male Mongolian gerbils (Meriones unguiculatus) and measured the layer-specific processing in the auditory cortex by tone-evoked current-source density (CSD) analysis. CSD analysis allows to investigate the synaptic population activity across the cortical depth and based on the spatiotemporal information flow to differentiate cortical layers I/II, III/IV, Va, Vb/VIa, and VIb ${ }^{18-20}$. We demonstrate that VTA stimulation effectuated a sensory gain amplification via thalamocortical inputs in the deep layers $\mathrm{Vb} / \mathrm{VIa}$, rather than via recurrent excitation in layer III/IV ${ }^{18,21}$. Our results demonstrate for the first time a functional diversification of the anatomically distinct thalamocortical input systems in the sensory cortex. Reward-modulated sensory input in deep layer neurons therefore might provide a cellular substrate for integrating sensory and task-related information in the service of sensory-based decision-making and reinforcement learning.

\section{Results}

Optogenetic stimulation of the VTA evokes self-stimulation behavior. To selectively target the excitatory projection neurons of the VTA, we expressed the red-shifted opsin C1V1 under the CamKII $\alpha$ promotor $^{22}$ by a stereotactically guided microinjection (Fig. 1A; $700 \mathrm{nl}$; AAV-CamKII $\alpha$-C1V1(E162T)-p2A-eYFP, $3 \mathrm{e} 12$ particles per $\mathrm{ml}$, UNC Vector Core). Opsin expression in the VTA overlapped significantly with tyrosine hydroxylase $(\mathrm{TH})$-immunostaining, selective for dopaminergic cell populations. The connectivity of the transduced neurons with the auditory cortex was confirmed by histology (Fig. 1A,B) in agreement with previous reports $^{6,23}$. After viral transduction, we waited 2-3 weeks for sufficient transgene expression before subjects were trained over the course of 10 days in an optogenetic intracranial self-stimulation task (Fig. 1C; Video S1; 10 pulses, $25 \mathrm{~Hz}, 473 \mathrm{~nm}$ laser-light per nose-poke; $10 \mathrm{~mW}$ power at fiber implant $)^{24}$. Animals that showed more than 50 presses/minute were chosen as the $\mathrm{C} 1 \mathrm{~V} 1$ group for electrophysiological experiments (Fig. $1 \mathrm{G})^{25,26}$. Anatomical validation of the fiber position relative to Bregma (AP: $-3.57 \mathrm{~mm}$, ML: $584.14 \mu \mathrm{m}$; cf. Figure 1D,E), as well as the fiber distance towards the area of opsin expression (Fig. 1F, $146.8 \pm 38.9 \mu \mathrm{m}$ ), was performed in a subset of animals $(\mathrm{n}=7)$ in the C1V1 group (see Materials and Methods). In contrast to the opsin group, animals of the YFP-control group showed no self-stimulation behavior (Fig. 1G).

Effect of VTA-activation on tone-evoked columnar activity in the auditory cortex. After evaluation of self-stimulation behavior in the C1V1 and YFP group, we performed acute laminar local field potential electrophysiological recordings from their auditory cortices under ketamine-xylazine anesthesia. This approach allows the investigation of circuitry effects by the stimulation of the VTA on sensory cortical processing. Additionally, we also recorded from a control group of naïve animals, which underwent no implantation or laser stimulation, to determine which effects were due to opsin activation or laser light ${ }^{27-29}$. Pure tones were presented in iso-octave steps in order to cover the main tonotopic range of the gerbil (Stimulation frequencies ranged from $0.125-32 \mathrm{kHz}$, duration: $200 \mathrm{~ms}$, inter stimulus interval 0.6-0.8 s, 50 repetitions, $65 \mathrm{~dB}$ SPL). After insertion of the recording electrode (NeuroNexus A1x32-50-413), pure tone measurements ( 7.5 minutes) were recorded over 45-75 min to guarantee stable recordings (Fig. 2A; see Materials and Methods). After the initial measurements, we paired the presentation of each pure tone within a single measurement ( 50 repetitions) with a laser-stimulation of the VTA $(473 \mathrm{~nm} ; 25 \mathrm{~Hz} ; 10 \mathrm{~ms}$ pulse length, $200 \mathrm{~ms}$ duration). Thereby, the VTA was stimulated 400 times within 7.5 minutes of the combined laser/tone presentation (Fig. 2). After this paired measurement, consecutive tone-only measurements (post) were performed over the course of 60 minutes. This procedure allowed us to observe phasic and long-lasting effects of the optogenetic VTA stimulation on ACx tone-evoked activity of all presented frequencies covering the range of the best frequency $(B F)$, near-BFs $( \pm 1-2$ octaves apart the $\mathrm{BF})$ and non-BFs $( \pm 3-4$ octaves apart the BF). We determined cortical BFs as the pure tone frequency, which evoked the highest peak amplitude within each layer in our recordings.

Figure $2 \mathrm{~B}$ displays a representative example of pure-tone evoked CSD-profiles after stimulation with the best frequency (BF, top) and near-BF (bottom, 2 octaves apart) before (left), during (center) and 30 minutes after (right) VTA- stimulation in a C1V1-transduced animal. Before VTA stimulation, tone-evoked CSD profiles show the canonical feed-forward information flow in sensory corte $\mathrm{x}^{30,31}$. Sink components with shortest onset latencies are related to thalamocortical input in cortical layers III/IV and the border between the infragranular layers V and $\mathrm{VI}^{18,19,32}$, described recently as $\mathrm{Vb} / \mathrm{VIa}^{19}$ (Fig. 2B, left). Subsequent later supra- and infragranular sink activities can be related to intracortical synaptic circuits in layers I/II, Va and VIb (see semi-automatic detection of sink activity in Materials and Methods and Figs. 2B and S2). Responses to frequencies apart from the BF generally evoke less prominent tone-evoked activity across all cortical layers. When pure tone stimulation is paired with VTA stimulation, we observed prominent changes of amplitudes, but not spatiotemporal flow of synaptic activity (Fig. 2B, center). This increase of tone-evoked cortical processing was long-lasting and persisted over approximately $30 \mathrm{~min}$ after the cessation of laser stimulation (Fig. 2B, right).

Laminar specificity of transient and long-lasting effects of VTA stimulation. In order to relate the observed changes of in current flow to distinct cortical layers, we separately analyzed CSD-traces from respective cortical layers (I/II, III/IV, Va, Vb/VIa and VIb; see Materials and Methods). Prominent sink components of cortical layers III/IV, Va, and Vb/VIa were related to early time windows of $0-50 \mathrm{~ms}$ after tone onset, while cortical layers I/II and VIb felt into later time windows of $>50 \mathrm{~ms}$ determined by sink onset (Fig. S2).

Described effects during and after VTA stimulation might impact on sensory processing in a less stimulus-locked manner and influence trial-by-trial signal-to-noise ratio ${ }^{33}$. In order to capture such variability, we have utilized a single-trial analysis using LME models ${ }^{34}$ on peak amplitudes of each layer as a function of time 

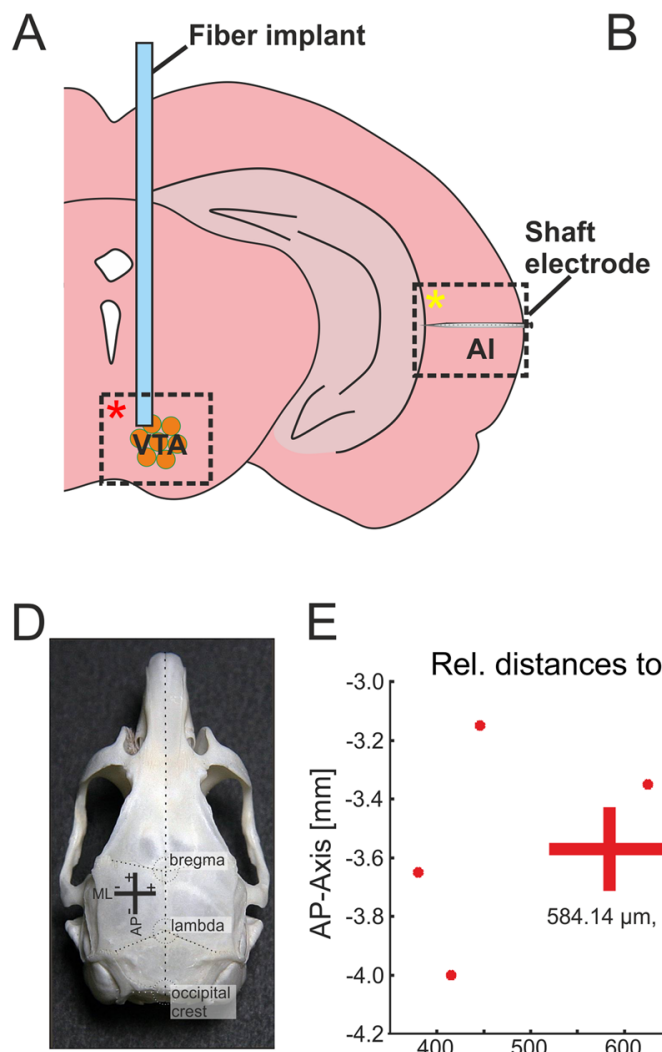

E

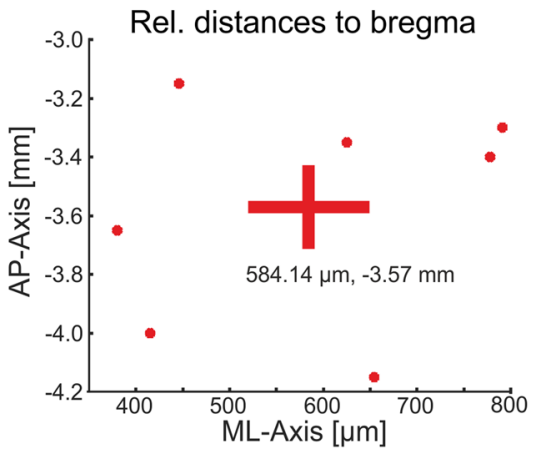

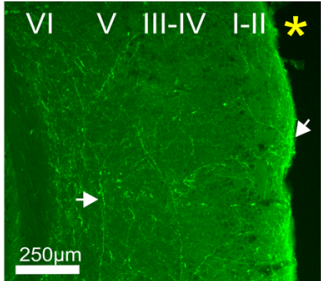

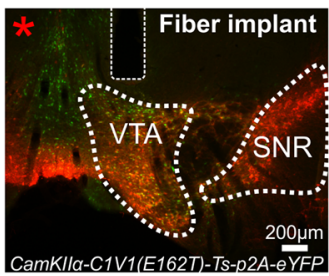

F
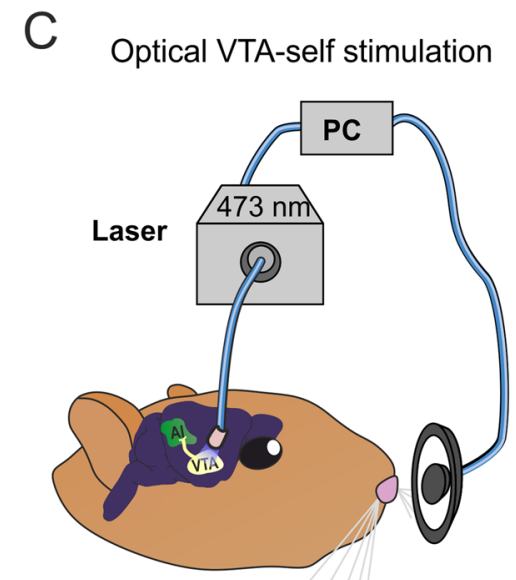

G

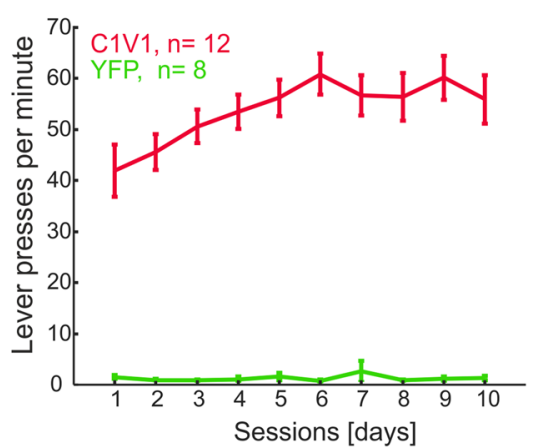

Figure 1. Anatomical validation of fiber position relative to target side and optogenetic intracranial selfstimulation (A) Schematic overview of fiber implant position for optogenetic stimulation of the VTA, and the ipsilateral AI recording side. Dashed boxes indicate representative regions of interest shown in B) identified by yellow and red asterisks. (B) Top, YFP expression in the temporal cortex covering the region of AI after virus transduction of the VTA indicates fiber terminals from the VTA targeting mainly upper layers and collaterals in deeper layers (white arrows). YFP expression was detectable in multiple regions of the neocortex (not shown). Bottom, co-fluorescence of YFP (AAV-CamKII $\alpha$-C1V1 (E162T)-p2A-eYFP) and TH-immunostaining (Alexa Fluor 546). Area of co-fluorescence is indicative of overlapping neuronal populations expressing the virus and TH mainly found in the VTA, and not the substantia nigra ${ }^{24}$. Scale bars indicate 250 and $200 \mu \mathrm{m}$, respectively. (C) Schematic representation of optogenetic intracranial self-stimulation: While nose-poking the lever, animals induce a light flash towards the VTA resulting in extensive self-stimulating behavior ${ }^{25}$. (D) Gerbil skull with prominent landmarks (bregma, lambda and occipital crest) for anatomical reference modified from Radtke-Schuller et al. 2016. For experiments, bregma has been used for reference of medio-lateral (ML) and anterior-posterior (AP) position of the VTA. (E) Estimated actual AP/ML coordinates with mean coordinates (AP:-3.57 mm; ML: $584.14 \mu \mathrm{m})$ relative to bregma of the C1V1group $(\mathrm{n}=7)$ as estimated with the gerbil brain atlas $^{84}$. (F) Fiber distance (mean \pm SEM) towards area of co-fluorescence $(146.8 \pm 38.9 \mu \mathrm{m} ; \mathrm{n}=7)$. Compare with bottom picture of $\mathrm{B})$. (G) Averaged lever pressing rates (lever presses/min) of the C1V1 $(\mathrm{n}=12)$ and YFP $(\mathrm{n}=8)$ groups over 10 days. A representative video of a C1V1 animal is accessible as Movie (See Video S1).

before and after VTA stimulation separately for BF, near- and non-BF stimulation for all groups (Fig. 3). Within each frequency bin, data was normalized to pre-measurements in order to characterize relative changes over time and compared both groups with laser stimulation (C1V1, YFP) against the control group. Within the control group, we observed a variation of tone-evoked responses of up to $\pm 10 \%$ over the entire recording time. Therefore, to obtain main effects of the laser stimulation and account for general variance, we quantified and statistically tested only data that crossed this $\pm 10 \%$ criterion of the normalized pre-measurements (see dashed lines in Fig. 3). In the C1V1 group, VTA stimulation led to a significant increase of peak amplitudes in infragranular layers Vb/ VIa most prominently after BF-stimulation for up to 45 minutes. The main current sink in thalamocortical input layers III/IV, in contrast, showed only minor modulation after VTA stimulation. Only 30 minutes after VTA stimulation near- and non-BF evoked activity was increased. Activity in layer Va was unaffected by VTA stimulation. Late onset sink components in layers I/II and VIb showed opposing temporal effects after VTA stimulation. While supragranular activity increased gradually and in a long lasting manner for $\mathrm{BF} /$ near- $\mathrm{BF}$ stimulation, infragranular layer VIb activity was most strongly amplified for non-BF only during combined tone-laser stimulation. In the YFP-group peak amplitudes of all sink components showed a general trend to be reduced during laser-stimulation and recovered over time within the $>45 \mathrm{~min}$ of post recordings. These effects were potentially due to light-related side-effects of the laser stimulation such as minimal temperature changes ${ }^{27-29,35}$, which might 


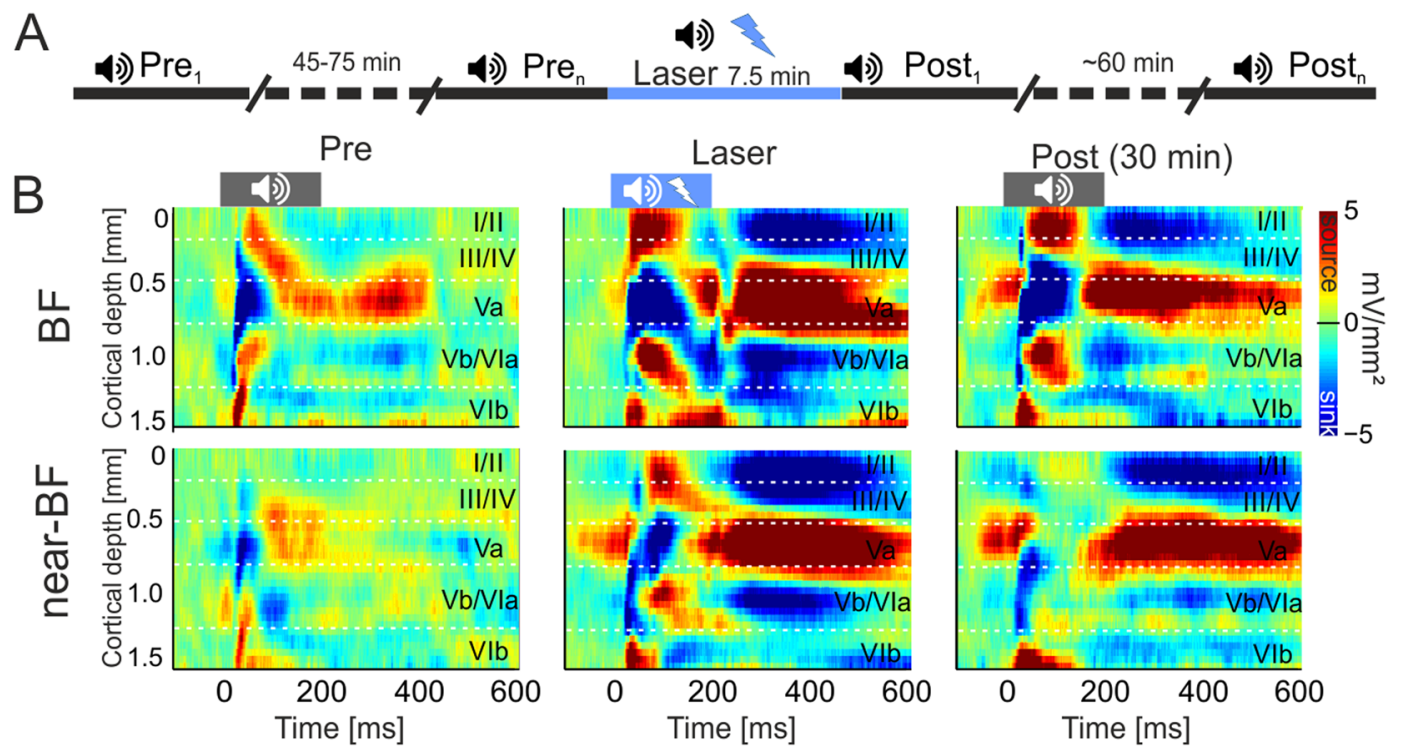

Figure 2. Electrophysiological measurements and effects of VTA stimulation on tone-evoked CSD profiles in AI (A) Schematic representation of the timeline of measurements. Pre baseline measurements (Tone stimulation: $0.125-32 \mathrm{kHz}$, tone duration: $200 \mathrm{~ms}$, ISI: $0.6-0.8,50$ pseudorandomized repetitions, $65 \mathrm{~dB}$ SPL, $7.5 \mathrm{~min}$ ) were performed until CSD patterns had stabilized ( $45-75 \mathrm{~min}$ ). A single measurement of combined tone and laser stimulation $(25 \mathrm{~Hz}, 473 \mathrm{~nm}, 10 \mathrm{~mW})$ was carried out and followed by a series of post-measurements (up to $60 \mathrm{~min}$ ). (B) Representative example of a CSD profile after BF- (top) and near-BF (bottom) stimulation before, during and 30 minutes after the combined tone/VTA-laser measurement from an animal of the C1V1 group. Before VTA stimulation, BF-evoked CSD profiles revealed a canonical feedforward pattern of stimulus-evoked current flow starting with initial sink components in cortical layers III/IV and Vb/ VIa. Subsequent sink activity was found in layers Va, I/II and VIb. Activity after near-BF stimulation showed a less pronounced columnar activation in the pre-condition. During paired tone and VTA stimulation, both stimulation frequencies evoked stronger and prolonged translaminar current flow, which was still enhanced 30 minutes later. Cortical layers are indicated. Dashed vertical lines indicate tone onset and offset.

shift the balance of excitation and inhibition in the $\mathrm{VTA}^{36-39}$. Data from control animals displayed a mainly stable amplitude range within the $\pm 10 \%$ range of pre-measurements.

Based on the most prominent changes of evoked activity in the C1V1-group during VTA stimulation (layer $\mathrm{VIb}$ ) and over the longer period of $>30 \mathrm{~min}$ after stimulation (layers $\mathrm{I} / \mathrm{II}$ and $\mathrm{Vb} / \mathrm{VIa}$ ), we compared slopes of evoked peak amplitudes as a function of stimulation frequency by linear fitting (Fig. 4). All data were normalized to BF-evoked amplitudes of pre-measurements within each layer separately. Slopes across the BF/near-BF/non-BF range therefore allow us to characterize changes in tuning sharpness. Frequency-independent gain increase, in contrast, would not affect the slope, but rather affect the intercept. In the pre-measurement, the most prominent frequency tuning was observed in thalamocortical input layers III/IV. In the pre-condition amplitudes in the non-BF bin were reduced about $60 \%$ compared to BF-evoked responses. Across frequency bins, this led to a slope of 0.28 indicating a significant dependence of amplitude and stimulation frequency (Fig. 4, dashed blue line, $\mathrm{p}<0.001$ ). During laser stimulation and 30 minutes after, slopes did not change (change of slope $>0.01 ; p>0.05$ ) indicative of a stable frequency tuning in layers III/IV. In layers Vb/VIa, a slope of 0.13 indicated a less prominent frequency tuning in the pre-condition. During VTA stimulation the frequency tuning was unaffected (change of slope $<0.01 ; \mathrm{p}<0.05$ ) but showed a significant increase of sharpness 30 minutes later (change of slope pre:30 min of $+0.05 ; \mathrm{p}<0.001)$. Comparably, in supragranular layers, I/II slopes of the pre-condition $(0.09 ; \mathrm{p}<0.001)$ did not significantly increase during VTA stimulation (change of slope $+0.04 ; \mathrm{p}=0.09$ ), but significantly increased $>30 \mathrm{~min}$ later (change of slope pre: $30 \mathrm{~min}+0.08 ; \mathrm{p}<0.001$ ). Hence, cortical layers $\mathrm{Vb} / \mathrm{VIa}$ and I/II showed no change of tuning bandwidth during the paired tone/VTA stimulation, but a significant increase in spectral integration prolonged for up to 30 minutes afterwards. Activity in layer VIb showed a prominent increase during VTA stimulation selectively for the non-BF bin (Fig. 3; bottom right), and, in accordance, we found slopes to be significantly decreased during laser stimulation (change of slope pre: laser $-0.06 ; \mathrm{p}<0.001$ ), which recovered after 30 minutes (change of slope Pre: $30 \mathrm{~min}-0.02 ; \mathrm{p}=0.10$ ). Layer Va similarly showed a decrease in slope during laser-stimulation (change of slope pre: laser $-0.04 ; \mathrm{p}<0.001)$, and also recovered 30 minutes later $(\mathrm{p}>0.05)$. VTA stimulation yielded a transient increase in response bandwidth in cortical layers Va and VIb.

Based on the layer-specific modulation of early tone-evoked inputs in thalamocortical-recipient layers $\mathrm{Vb} / \mathrm{VIa}$ and subsequent synaptic inputs in supragranular layers, we speculate that stimulation of the VTA affects early and late sensory processing on a local and corticocortical level, respectively ${ }^{17}$.

Impact on local intracolumnar and corticocortical synaptic inputs. In a next step, we therefore aimed to disentangle early activity associated with local thalamocortical input, and subsequent activity associated 
BF
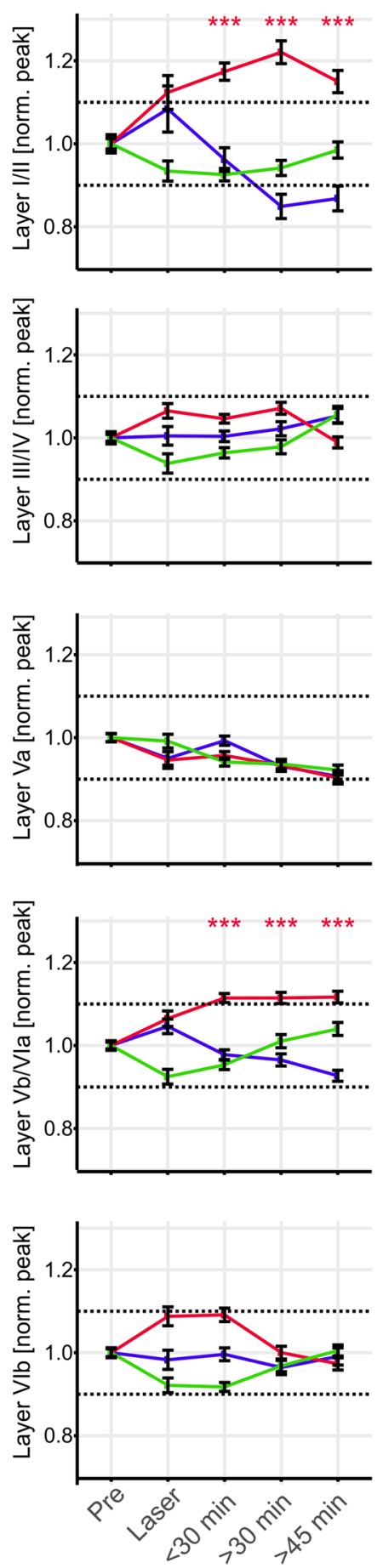

near-BF
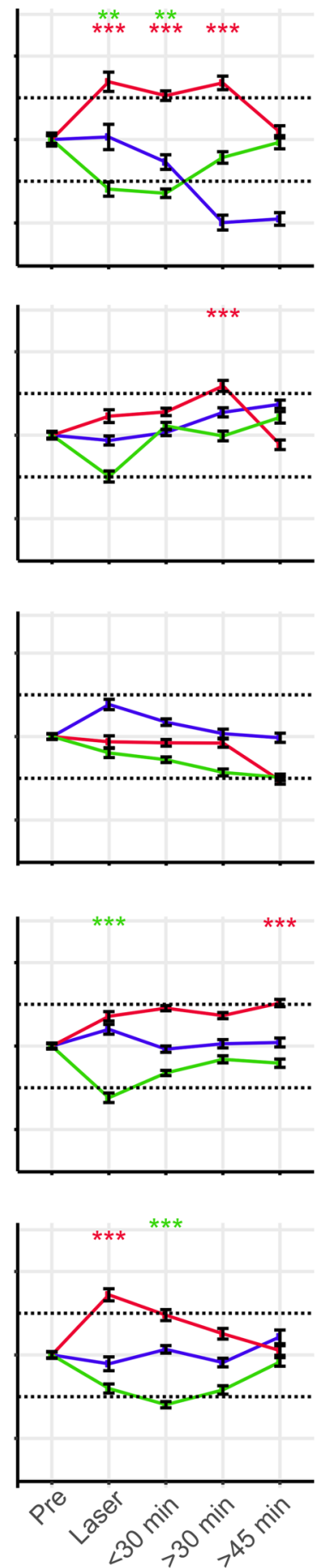
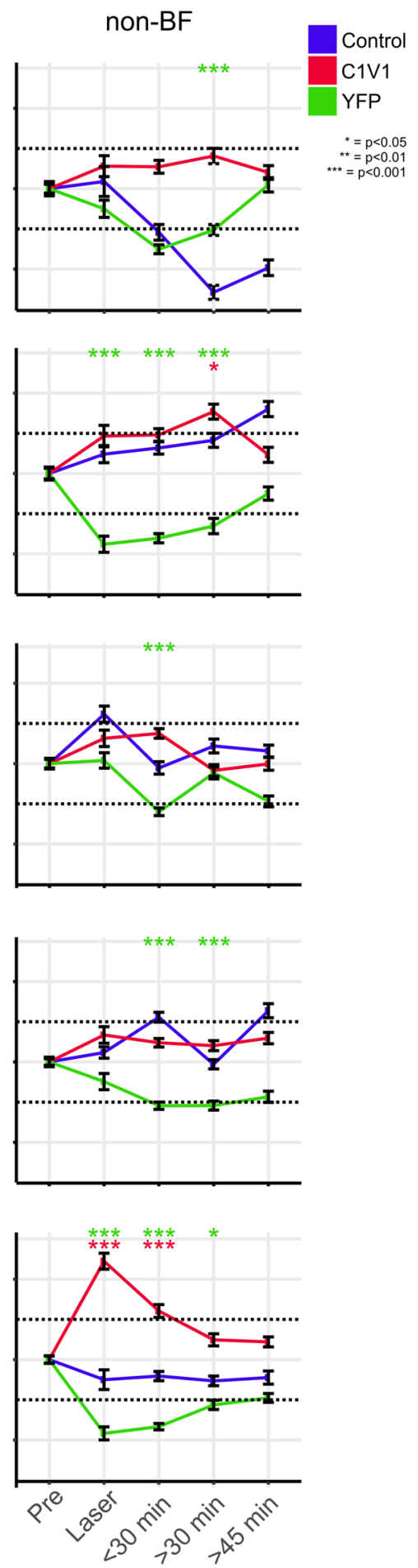

Figure 3. Binned time courses of single-trial sink peak amplitude data of layer-specific CSD-traces Temporal development of single-trial sink peak amplitudes for early sink activity (sink onset $<50 \mathrm{~ms}$ ) in layers III/IV, $\mathrm{Va}$ and $\mathrm{Vb} / \mathrm{VIa}$ and late sinks (sink onset $>50 \mathrm{~ms}$ ) in layers $\mathrm{I} / \mathrm{II}$ and VIb for BF, near- and non-BFs. Layer I/ II peak amplitudes showed a significant increase for the C1V1 group during laser and in post-measurements, surpassing the $+10 \%$ criterion within the BF and near-BF bins. Significant differences from the control group are again revealed by LME models and indicated by asterisks. Layer I/II peak amplitudes in the YFP and control group did not show corresponding increases over the recording time but showed a decrease and recovery for the YFP group. Layer III/IV activity did not reveal differences over time for BF-evoked responses in all groups. With spectral distance from the $\mathrm{BF}$ the $\mathrm{C} 1 \mathrm{~V} 1$ group showed a moderate increase yielding significance at the $>30 \mathrm{~min}$ time bin compared to control animals. Within the non-BF bin, peak amplitudes showed a significant decrease in the YFP group during and after laser stimulation. Layer Va peak amplitudes were most stable across time and groups only displaying a significant decrease for the YFP group in the non- $\mathrm{BF}$ bin a $<30 \mathrm{~min}$. Layer $\mathrm{Vb} / \mathrm{VIa}$ peak amplitudes in the C1V1 group were significantly increased in all post-measurements after BF-stimulation. 
For stimulation with near-BF a similar trend was found. Significant changes between the YFP and control group were again due to decreased peak amplitudes in the YFP group. Layer VIb displayed a highly significant increase in the $\mathrm{C} 1 \mathrm{~V} 1$ group during laser stimulation, most prominent in the non-BF bin, and less prominent in near-BF and BF bins. Laser-induced increase only persisted for the next time bin, but then recovered to pre-condition. While the control group showed stable peak amplitudes over the time course, YFP animals again showed a moderate decrease most prominent in off-BF bins.

with corticocortical processing. We compared changes in the tone-evoked average rectified CSD (AVREC) and the residuals of the CSD (ResidualCSD) during early (0-50 ms) and late ( $80-300 \mathrm{~ms})$ time windows (Fig. 5A). This rationale is based on previous findings demonstrating that the residual CSD reflects the spatiotemporal ratio of unbalanced sinks and sources providing a quantitative measure of horizontal intercolumnar inputs to a given cortical site ${ }^{18,20}$. The AVREC, on the other hand, reflects the overall intracolumnar current flow at a given recording site ${ }^{31,40}$. Figure 5A (left) shows changes of both the grand average of the AVREC (top) and relative CSD (bottom) waveforms before, during, and, in certain time windows, after VTA stimulation in the C1V1 group. Comparable changes were not observed in neither the YFP nor control group (Fig. S3). To further investigate temporal changes of BF-evoked responses in more detail, time course of binned root mean square (RMS) values for early and late averaged and normalized AVREC and ResidualCSD windows of each group were plotted as a function of time (normalized to pre-measurements). RMS values of the AVREC during the early phase (0-50 ms) displayed a significant increase in the C1V1 group from $15 \mathrm{~min}$ until $52 \mathrm{~min}$ after VTA stimulation. Significant effects were validated with a LME model (Fig. 5B; $\mathrm{p}<0.001-0.025)$. During the late phase (80-300 ms) after tone onset, RMS of the averaged AVREC did not show any significant changes between each group (Fig. 5B). During laser stimulation, a significant increase has only been observed for the averaged early ResidualCSD indicative of amplified lateral contributions to early tone-evoked columnar activity in the C1V1 group $(\mathrm{p}=0.04)$. Furthermore, early and late ResidualCSD were both significantly enhanced during later time points after the VTA stimulation (early ResidualCSD: 22.5-37.5 min; $\mathrm{p}=0.014-0.039$; late ResidualCSD: $37.5-45 \mathrm{~min}, \mathrm{p}=0.008-$ 0.033). Averaged RMS tuning curves of the AVREC and ResidualCSD of the C1V1 group revealed an increase of tone-evoked activity during and after laser stimulation across the entire range of BF, near-BF $( \pm 1-2$ octaves $)$ and non-BF ( $\pm 3-4$ octaves) stimulation (Fig. $5 \mathrm{C}$ ).

Integration of local and corticocortical synaptic inputs in dependence of the stimulation frequency. All single-trial AVREC and ResidualCSD RMS values were normalized to pre measurements. Normalized values were analyzed as a function of time before and after VTA stimulation binned for BF, near-, and non-BF stimulation for all groups (Fig. 6). Figure 6A (top) shows a gradual increase of the early AVREC compared to the control- and YFP-group independent of stimulation frequency. For BF- and near-BF-stimulation, this yielded a significant increase at $>30 \mathrm{~min}$ after VTA stimulation. The ResidualCSD also showed a strong increase almost independent of the stimulation frequency already during the combined tone-laser presentation and all following post measurements. Consistently with the layer-specific activity, there was a less pronounced decrease of the near/non-BF-evoked AVREC in early and late time windows in the YFP-group during laser stimulation, which started to recover over time (Fig. 6A, bottom).

In the later time window of 80-300 ms after tone-onset, only the ResidualCSD showed a significant increase during and up to 30 minutes after VTA stimulation (Fig. 6B). Altogether, VTA stimulation led to a gradual increase of the overall early current flow most pronounced for BF- and near-BF stimulation. This effect was long-lasting and peaked around 30 minutes after stimulation. We further found an immediate and long-lasting increase of imbalance between sinks and sources based on the early and late ResidualCSD in all frequency bins indicative of amplified corticocortical activity.

\section{Discussion}

The VTA is a key structure to convey information about stimulus salience (conspicuity) and value (context) to target areas distributed throughout the brain in the awake individual ${ }^{2}$. Receiving both sensory as well as VTA inputs, the primary sensory cortex is ideally positioned to integrate such bottom-up and top-down information to shape behavior through reinforcement learning. We here demonstrate for the first time that activation of VTA-reward circuits amplifies bottom-up sensory input via a yet largely overlooked thalamocortical projection targeting infragranular layers $\mathrm{Vb} / \mathrm{VIa}$ of the auditory corte $\mathrm{x}^{32,41}$. In contrast, granular layer III/IV input, a well-known substrate for sensory gain modulation ${ }^{18,21,41,42}$, was unaffected by VTA stimulation. The impact of the observed infragranular gain modulation on supragranular layers manifests as a translaminar long-lasting enhancement of frequency tuning (see Fig. 7). Furthermore, we demonstrate that the frequency-specific gain in deeper layers emerges on a local columnar level and yields an increased corticocortical integration displayed in lateral input most likely terminating in upper layers. This highlights the diverse, and yet elusive functions of cortical layers in the sensory cortex ${ }^{43}$. The long-lasting modulation after VTA stimulation reveals that the influence on sensory cortex transcends the time scales commonly associated with reward-prediction error coding.

The optogenetic stimulation of VTA-projection neurons allowed us to investigate effects of reward-related cross-regional circuits (Fig. 1) ${ }^{25}$. Roughly $8 \%$ of non-sensory inputs of gerbil ACx arise from direct projections of the ipsilateral VTA, which terminate across the cortical column in a layer-specific manner ${ }^{6,13}$. Direct stimulation of the VTA projection neurons is an effective method to activate this circuit and evaluate its particular impact on the cortical bottom-up processing. While optogenetic terminal stimulation in the cortex would be an even more direct way to trigger transmitter release from these fibers locally, the method is complicated, however, by back-propagating action potentials and the highly inhomogeneous illumination in a relatively thick structure 


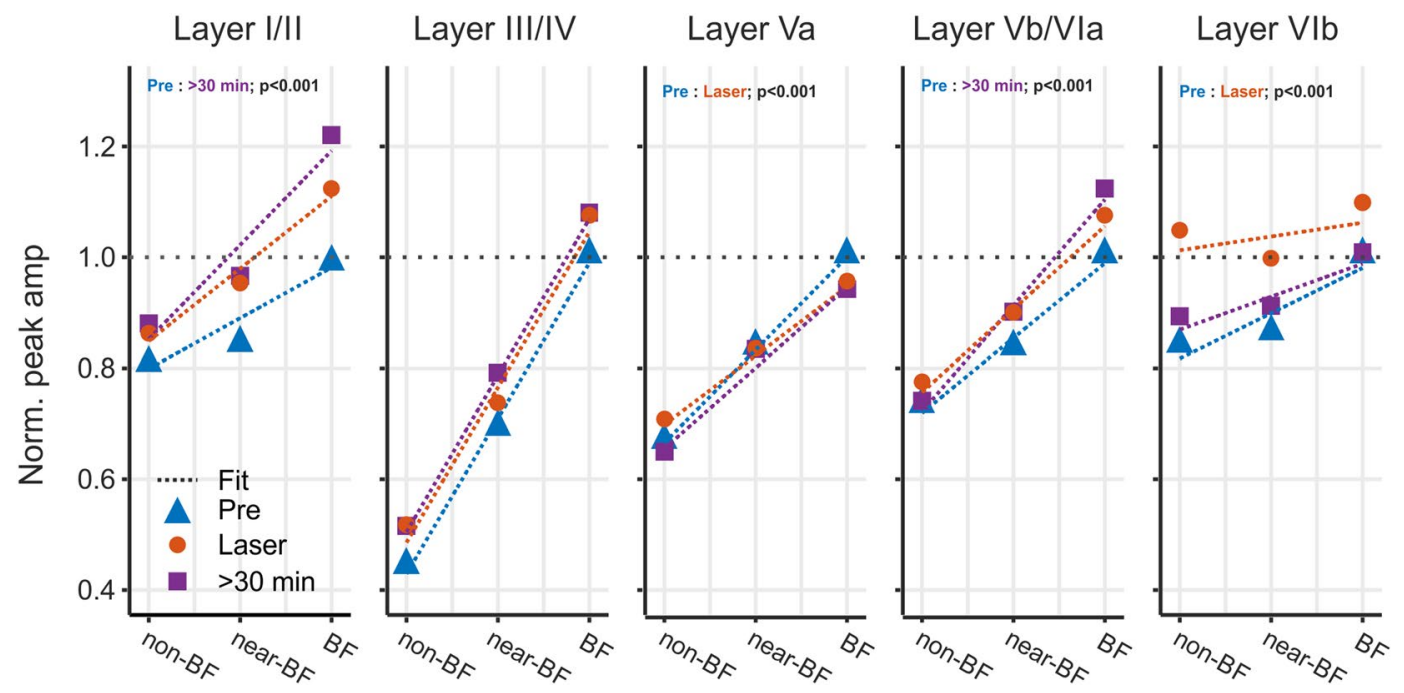

Figure 4. Layer-specific early and late changes of tuning sharpness of the C1V1 group For data from the C1V1 group, peak amplitudes within each layer were normalized to BF-responses before laser stimulation. We fitted a linear fit (dashed lines) across evoked responses across the BF-, near-BF- and non-BF-bins and calculated the slope as indicator for spectral tuning sharpness (for pre, laser, $>30 \mathrm{~min}$ ). Sharpest tuning properties of peak amplitudes were found in layers III/IV (slope of 0.28). Tuning in layers III/IV was not affected by VTA stimulation. Layers I/II showed less prominent tuning revealed by a shallower slope in the pre-condition. While VTA stimulation did not yield an immediate significant difference, slopes indicated a significantly sharper tuning $>30$ min after VTA stimulation $(\mathrm{p}<0.01)$. This was due to mainly an increase in BF-evoked peak amplitudes in accordance with Fig. 5. Peak amplitudes in infragranular layers Vb/VIa showed a similar effect as layers I/II with a trend of sharper tuning during VTA stimulation that yielded significance $>30$ min later. In contrast, only in late infragranular layer VIb, peak amplitudes revealed a significantly shallower slope indicative of a broader frequency tuning during laser stimulation (Pre: laser; $\mathrm{p}<0.01$ ), which recovered after $>30 \mathrm{~min}$ (Pre: $<30 \mathrm{~min} ; \mathrm{p}=0.1$ ). This can be seen by the flat slope between non- and BF bins. Layer Va only showed a minor decrease of the slope during VTA stimulation that recovered partially $>30 \mathrm{~min}$ later. Significances indicated are based on slope comparisons performed using the Ismeans package in R (version 2.27-62). For statistical explanation, see text.

such as the cortex. Due to our emphasis on phenomena at the laminar processing level, VTA stimulation provides an activation of dopaminergic fibers across the different cortical layers due to the actual functional anatomy of the interregional circuits. Furthermore, due to controversial findings about direct heat-related effects on neuronal activity ${ }^{27,29,35}$, the remote stimulation of the VTA allows for a more homogenous manipulation of processing across cortical layers.

VTA stimulation led to increased early tone-evoked $(0-50 \mathrm{~ms})$ local columnar current flow and increased long-range corticocortical activity. This effect persisted for up to 50 minutes after VTA stimulation and was most prominent for the respective BF of the given recording site (Fig. 5). Hence, VTA stimulation promotes the local gain of columnar input over a prolonged period and lead to substantial corticocortical integration of spectral information. Notably, the gain amplification of sensory input appeared exclusively in thalamocortical-recipient layers $\mathrm{Vb} / \mathrm{VIa}$ but was absent in the main thalamocortical-recipient granular layers III/IV (Fig. 3). This enhancement of infragranular activity was locally relayed to supragranular layers I/II. This mechanism may provide a way for intratelencephalic neurons in infragranular layers ${ }^{44}$ to provide a translaminar gain control of sensory representation depending on the experience of reward ${ }^{45,46}$. Although direct projections of the ipsilateral VTA provide a significant contribution to non-sensory inputs of the gerbil $\mathrm{ACx}^{6}$, our approach may recruit other relay stations that could contribute to the observed effects ${ }^{5}$. Further, dopaminergic VTA projections to subcortical earlier auditory pathway stations, like the auditory thalamus, are reported to be only very scarce ${ }^{47,48}$. In accordance, no significant changes were observed for the dominant thalamic input sink in layers III/IV supporting the mainly intracortical origin of the described effect.

Previous reports investigating effects of dopamine on tuning sharpness in the $\mathrm{ACx}^{4,49}$ raised the question how reward information modulates the representation of spectral information on a circuit level. Our data showed that stimulating the VTA projections only affected early auditory tuning sharpness in deep cortical layers, but not in the main thalamocortical input layer III/IV ${ }^{41,50}$. Sharper frequency representation was relayed to superficial layers I/II and persisted over time after VTA stimulation. Our observations are thereby consistent with recent findings from layer-specific high-field fMRI in human auditory cortex showing sharper tuning in upper layers depending on attention and task engagement ${ }^{51}$.

As a result, reward-circuit activation promotes a long-lasting gain enhancement of salient auditory stimuli via strengthened infragranular thalamocortical inputs and increased sensory integration in supragranular circuits. Our finding now provides an explanatory role of the anatomically and physiologically well-described early infragranular inputs in layers $\mathrm{Vb} / \mathrm{VIa}$ of the sensory cortex ${ }^{18,32,41,52}$ as a circuit for integrating sensory and task-related information. 
A

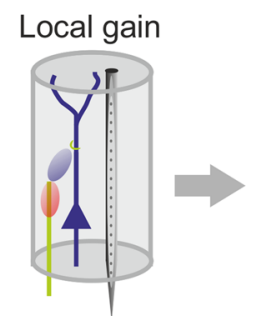

Corticocortical
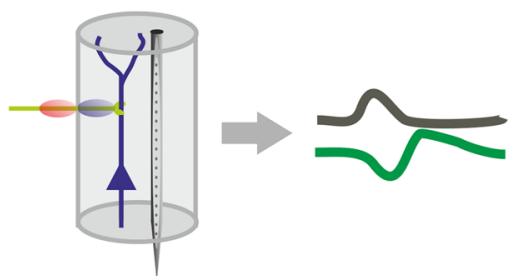

B

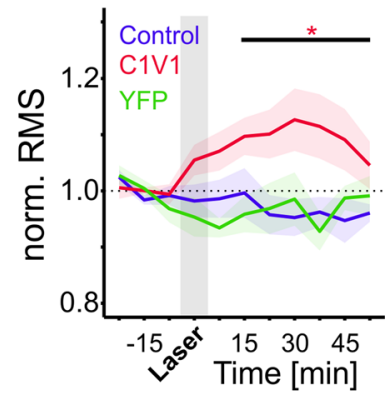

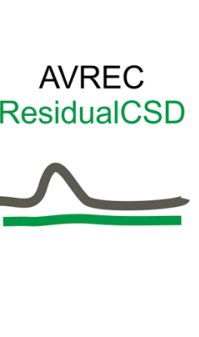
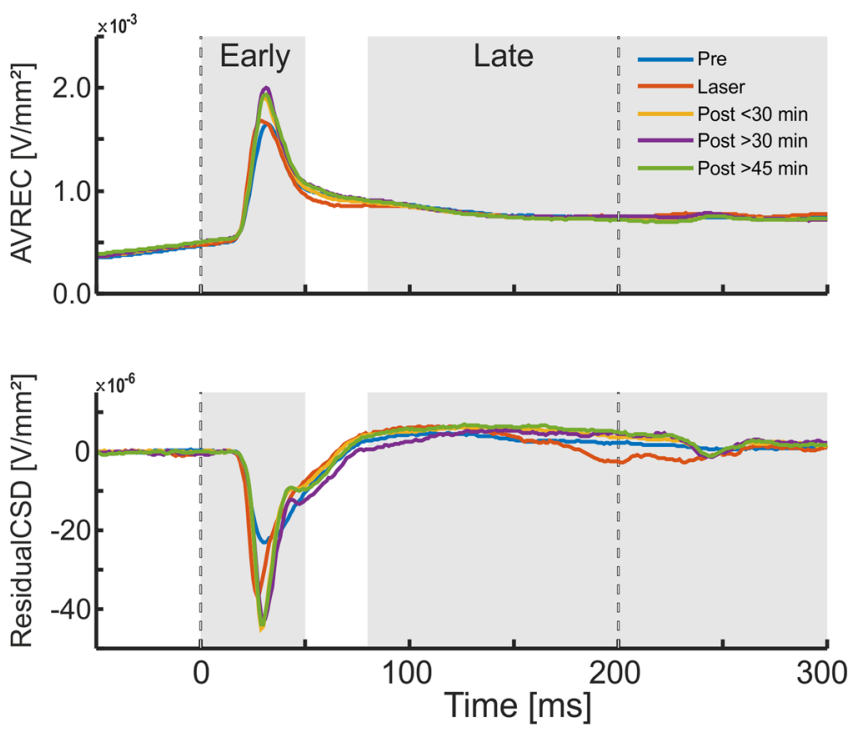

C

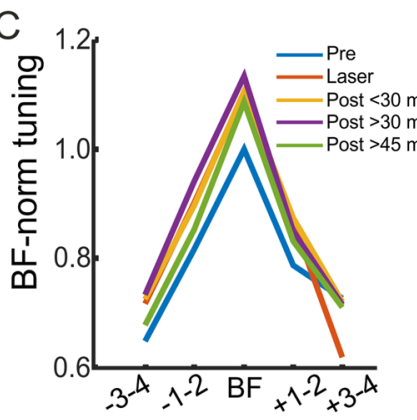

Binned frequency [oct.]
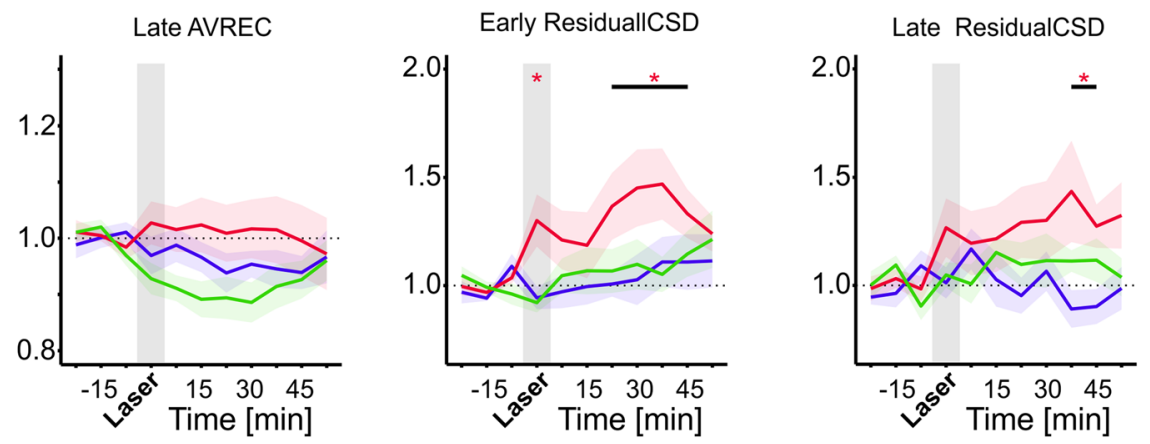

Figure 5. Temporal development of early and late AVREC and ResidualCSD activity (A) Grand average traces of the BF-evoked AVREC (top) and ResidualCSD (bottom) of the C1V1 group $(\mathrm{n}=12)$ for binned pre, laser and post $(<30,>30 \&>45 \mathrm{~min})$-measurements. Right, insets illustrate that AVREC activity is mainly associated with local columnar activity, while the ResidualCSD quantifies contributions from lateral corticocortical input (cf Happel et al., ${ }^{18,20}$ ). (B) Temporal time courses of Pre-normalized early/late AVREC and early/late ResidualCSD traces of C1V1 (red), control (blue) and YFP (green) groups ( $=12, n=7, n=7$, respectively) shown for BF stimulation. Early AVREC signal displayed a significant increase in post-measurements for the C1V1 group whereas time courses of the control and YFP groups were stable. Late AVREC signal showed no significant differences between either group. RMS ResidualCSD values of early and late time windows displayed a similar temporal development as the early AVREC becoming significantly increased in the later postmeasurements within the C1V1 group. Control and YFP groups showed no significant changes over time. Note the peaking in the early ResidualCSD RMS during laser stimulation, which is only present in the C1V1 group. Asterisks mark significant changes according to the applied mixed linear models of the normalized data towards the control group (see Materials and Methods). (C) Tuning curves of the RMS value normalized to BF-evoked responses in the pre-condition for early/late AVREC and early/late ResidualCSD for binned time points for C1V1 group. Note the general increase of the overall tuning curve relative to pre measurements. 
A
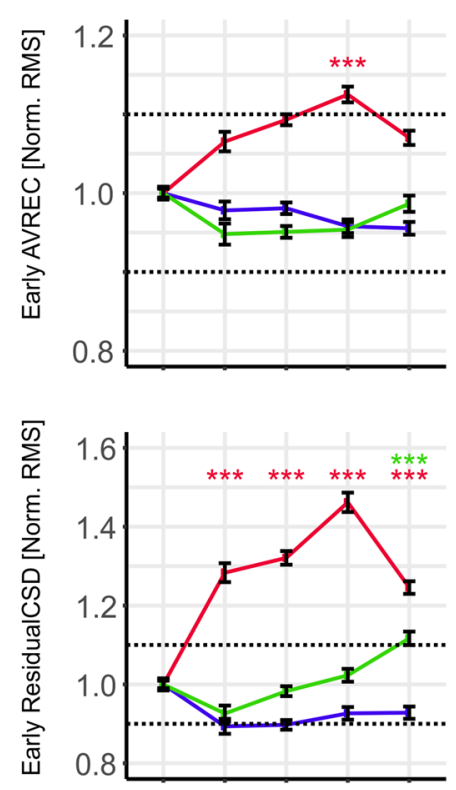

B
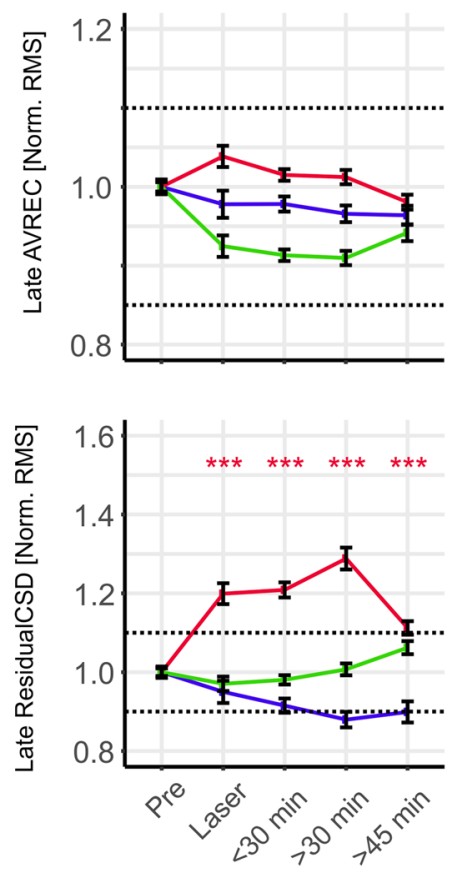

near-BF
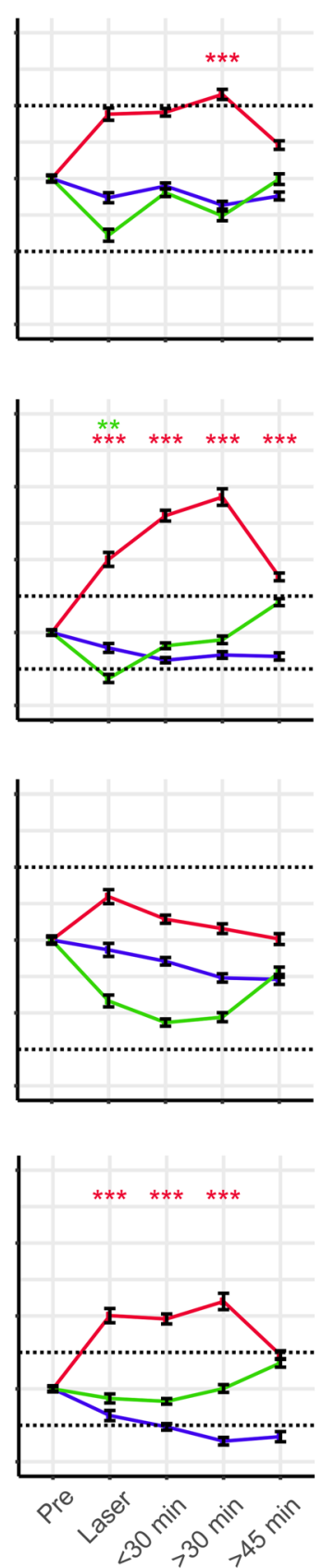

non-BF
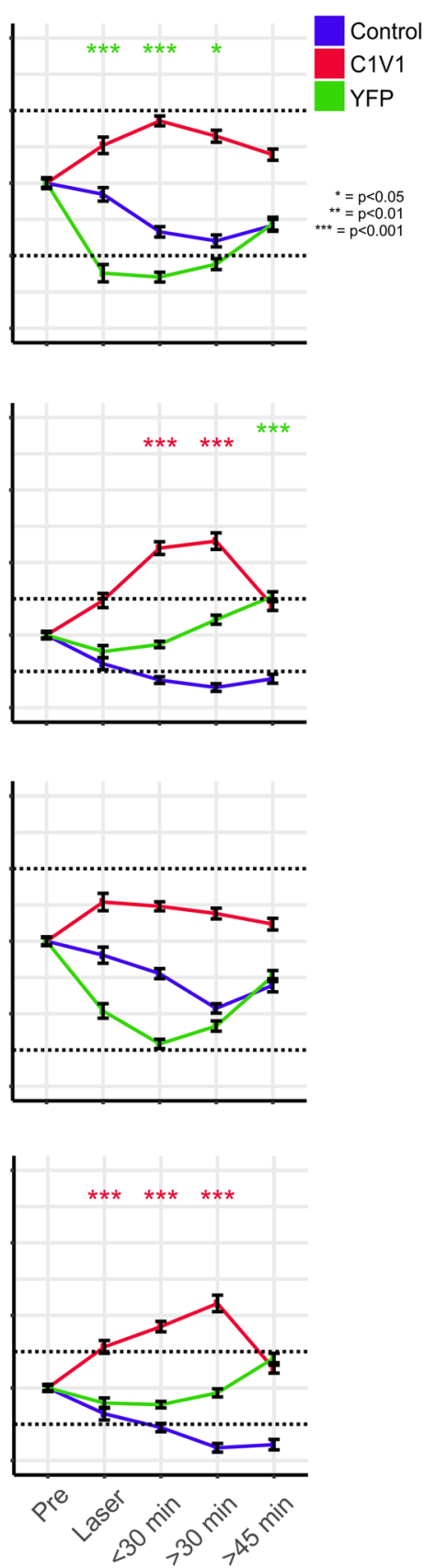

Figure 6. Normalized single-trial early and late AVREC and ResidualCSD RMS values over time. (A) Temporal time course of averaged early single-trial RMS AVREC (top) and ResidualCSD (bottom) values normalized to pre-measurements. Data for BF, near-BF and non-BF stimulation is shown separately and was binned for premeasurements, laser, and post-measurements $(<30 \mathrm{~min},>30,>45 \mathrm{~min})$. Note the prominent increase of early RMS AVREC values for the C1V1 group whereas control and YFP group displayed a more stable or decreasing time course, respectively. Also, early RMS ResidualCSD values significantly increased during and after the Laser measurements for BF, near- and non-BF bins of the C1V1 group, whereas YFP and control display a more stable time course. (B) Temporal time course of late Pre-normalized RMS AVREC and ResidualCSD values of singletrial data for BF, near-BF and non-BF. Late RMS AVREC values for the C1V1showed a mild, yet not significant, increase that did not surpass the $+10 \%$ criterion. The control group displayed a stable and slightly declining time course for the late RMS AVREC values, while the YFP group showed a moderate decrease that recovered over time. Late RMS ResidualCSD values increased during and after the Laser measurements for BF, near- and non-BF bins of the C1V1 group, whereas YFP and control groups both showed a more stable time course. Significances in $(\mathbf{A}, \mathbf{B})$ were calculated using mixed linear models on the normalized data. Asterisks indicate corresponding significances in comparison to the control group, in case values exceed the $\pm 10 \%$ pre-criterion. Single-trial CSD data in following figure is presented equally. 


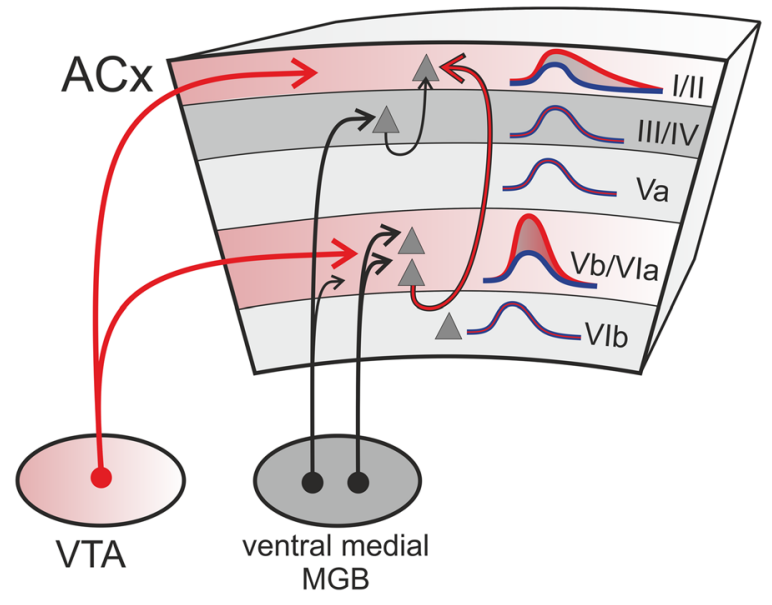

Figure 7. Schematic illustration of convergent deep layer inputs from sensory thalamus and VTA. VTA projections mainly target upper and infragranular layers of the gerbil auditory cortex (see also Fig. 1B). Stimulating the VTA projections led to a frequency-specific gain amplification of early sensory-evoked responses in thalamocortical recipient layers $\mathrm{Vb} / \mathrm{VIa}$. This gain increase effectuated an enhanced spectral tuning representation in supragranular layers I/II. The supragranular gain modulation might hence be inherited via intratelencephalic neurons targeting upper layers and bypassing granular input circuits ${ }^{44}$, may originate from a direct influence of dopamine released in upper layers or a combination of both. MGB, medial geniculate body.

Recent reports have highlighted that sensory-evoked responses in deep layers are modulated by behavioral states $^{53,54}$ and undergo substantial learning-related plasticity ${ }^{55}$ not described for middle layer thalamocortical input $^{56}$. Our data now assigns a functional impact of the VTA on deep cortical layers-particularly under the control of dopaminergic neuromodulation (Fig. 7).

Optogenetic VTA stimulation preferentially recruits the main output population of dopaminergic projection neurons making up roughly $55-65 \%$ of all VTA neurons ${ }^{57-59}$. Nevertheless, it has been demonstrated that the small population of non-dopaminergic projection neurons also convey reward information ${ }^{60-62}$ and particularly promote the activation of brain-wide regions including the auditory corte ${ }^{25}$. Furthermore, there is overlap between dopaminergic and glutamatergic cells through transmitter co-release ${ }^{63-66}$. Nevertheless, the long duration of responses modulation in our data set strongly argues for a significant dopaminergic component, as similar accumulative effects of dopamine have been shown in other studies ${ }^{11,67}$, whereas glutamatergic effects are generally more transient ${ }^{68,69}$. Specifically Lavin et al. (2005), showed that a single instance of electrical burst stimulation within the VTA transiently increases the spiking rates within neuronal populations of the prefrontal cortex over a time course of more than 30 minutes in close accordance to the temporal effects found in our study. This effect was not observed during the presence of various dopaminergic antagonist including sulpiride, SCH23390 and reserpine $^{67}$. Further evidence for a dopaminergic origin of the long-lasting effects is provided by the distribution of dopamine receptors, which, are concentrated mainly in infragranular and supragranular layers of the gerbil ACx where we see modulatory effects (Fig. 1B) ${ }^{13,70-74}$.

Phasic release of dopamine from the ventral tegmental area has long been associated with the reward prediction error signal postulated by reinforcement learning theory. How dopamine released by the VTA affects the plasticity that integrates sensory information and such learning-related signals in sensory cortex, however, is unknown. Our findings therefore assign a specific function to this rather understudied cortical circuit: VTA-modulated input to deep layer neurons in the sensory cortex mediates the integration of sensory and task-related information even when they are not precisely temporally contingent. Our study pursues the idea of a VTA-mediated local gain of sensory input at infragranular thalamocortical terminals via presumably presynaptic and metabotropic dopamine receptors ${ }^{17,67,75,76}$. Previous studies have described accumulative levels of dopamine in the auditory cortex over 30 minutes after training onset ${ }^{11}$ suggesting a long-lasting action of dopamine at the presynaptic terminals ${ }^{66}$. Furthermore, prolonged activation of D1/D5 receptors has been linked to mTOR-mediated and protein-synthesis dependent synaptic plasticity ${ }^{13}$ potentially inducing for instance proteolysis-dependent digestion of the scaffolding extracellular matrix around synaptic contacts ${ }^{77}$. Thereby, the here described microcircuit may promote the re-organization of the cortical 'bottom-up' tonotopic map by association of a specific pure tone frequency and an artificial 'top-down' modulation by stimulation of the VTA in anesthetized and awake animals over time $e^{4,5}$. We propose that the sensory gain amplification within deep layer neurons in the sensory cortex described in this study may potentially promote a sustained perceptual salience or value coding of incoming stimuli ${ }^{78,79}$, which our study is, however, not able to further differentiate ${ }^{80-82}$. Our findings now provide a circuit level explanation of the necessary integration of sensory and task-related information even when they are not precisely temporally contingent. Thereby, this circuit might constitute a substrate for the credit assignment in reinforcement learning theory concerned with the temporal delay between sensory input and its behavioral consequences ${ }^{83}$. 


\section{Methods}

All experiments were carried out in adult male Mongolian gerbils (Meriones unguiculatus, age 4-8 months, body weight: $70-100 \mathrm{~g} ; \mathrm{n}=26)$. The C1V1 group $(\mathrm{n}=12)$ consisted of animals that were expressing the viral construct, allowing for optogenetic stimulation of the VTA whilst recording. The control group $(n=7)$ consisted of non-transfected animals to control for temporal stability of the signal. In order to investigate effects of the laser-stimulation itself, a YFP-control group $(n=7)$ was included. Experiments were conducted in accordance with ethical animal research standards defined by the German Law and approved by an ethics committee of the State of Saxony-Anhalt.

Viral transduction of the VTA. Gerbils were anesthetized by an initial intraperitoneal injection $(0.005 \mathrm{ml} / \mathrm{g}$ BW) of $45 \% \mathrm{v} / \mathrm{v}$ ketamine ( $50 \mathrm{mg} / \mathrm{ml}$, Ratiopharm $\mathrm{GmbH}$ ), $5 \% \mathrm{v} / \mathrm{v}$ xylazine (Rompun, $2 \%$, Bayer Vital GmbH) and $50 \% \mathrm{v} / \mathrm{v}$ of isotonic sodium-chloride solution ( $154 \mathrm{mmol} / \mathrm{l}, \mathrm{B}$. Braun AG). Level of anesthesia was steadily checked by checking the paw-withdrawal reflex on a regular basis and additional doses of ketamine solution were applied if applicable $(0.06-0.08 \mathrm{ml} / \mathrm{h}$ ). Animals were fixed in an automated stereotaxic injector (Neurostar) for virus transduction ( $700 \mathrm{nl} ; 100 \mathrm{nl} / \mathrm{min} ; 5 \mathrm{~min}$ resting; C1V1: AAV5-CamKII $\alpha-C 1 V 1(\mathrm{E} 162 \mathrm{~T})-\mathrm{p} 2 \mathrm{~A}-\mathrm{eYFP}$ or YFP: AAV2-CamKII $\alpha$-eYFP 3E12 particles/ml, UNC Vector Core; target side: AP: - 3.8--4.0; ML: 0.5; DV: -6.2 mm, bregma for reference) and fiber implantation with a planar setting of bregma and lambda. Custom made fiber implants (fiber implant length $8 \mathrm{~mm}, \varnothing 230 \mu \mathrm{m}$, NA 0.39) were implanted at target side (AP: -3.8--4.0; ML: 0.5; DV: $-5.8 \mathrm{~mm}$ ). Fiber positions were determined by histological analysis and compared with the gerbil brain atlas (Fig. 1D,E) ${ }^{84}$. Animals were kept for 3 weeks to allow recovery and enough protein expression.

Intracranial self-stimulation using optogenetic VTA activation. Successful activation of the VTA through optogenetic stimulation was confirmed in an intracranial self-stimulation paradigm. Animals were trained for 10 consecutive days (at $20 \mathrm{~min}$ per day). By activating a lever with their nose, they could elicit brief laser stimulation (10 pulses, $25 \mathrm{~Hz}, 473 \mathrm{~nm}, 10 \mathrm{~mW}$ ) delivered to the VTA through the implanted fiber (Fig. 1A-C). Only animals pressing the lever more than 50 presses/minute were kept for the C1V1 group (Fig. $1 \mathrm{G})^{24-26}$.

Electrophysiological recordings. Anesthesia was initiated and controlled as described above during viral transduction of the VTA. The right auditory cortex was exposed by trepanation and target location of AI was chosen based on vascular landmarks. Body temperature was kept stable at $34^{\circ} \mathrm{C}$. Due to an epileptic seizure during the electrophysiological recordings we had to omit an animal from the YFP group.

Experiments have been carried out in an electrically shielded, soundproof chamber. The animal was head-fixed $1 \mathrm{~m}$ away from the speaker (Tannoy arena satellite KI-8710-32) by screwing the head post to a custom-made holder. Local field potentials (LFPs) have been recorded with a linear 32-channel shaft electrode (NeuroNexus A1x32-50-413), which was implanted perpendicular to the surface area of $\mathrm{AI}^{18}$. LFPs have been pre-amplified 500 -fold and band-pass filtered $(0.7-300 \mathrm{~Hz})$ with a PBX2 preamplifier (Plexon Inc.). Data were digitized at a sampling frequency of $1 \mathrm{kHz}$ with a multichannel recording system (Multichannel Acquisition Processor, Plexon Inc.).

Pure tones spanning 8 octaves (frequency range between $125 \mathrm{~Hz}-32 \mathrm{kHz}$, tone duration: $200 \mathrm{~ms}$, inter stimulus interval: $0.6-0.8 \mathrm{~s}, 50$ pseudorandomized repetitions, $65 \mathrm{~dB}$ SPL, $7.5 \mathrm{~min}$ per measurement) were generated in MATLAB, converted into an analog signal by a data acquisition card (NI PCI-BNC2110, National Instruments, Germany), rooted through a controllable attenuator (gPAH, Guger, Technologies, Austria) and amplified by an audio amplifier (Thomas Tech Amp75). A measurement microphone and conditioning amplifier were used to calibrate acoustic stimuli (G.R.A.S. 26AM and B\&K Nexus 2690-A, Bruel \& Kjaer, Germany).

Electrophysiological measurements were performed according to the scheme of Fig. 2A. After implantation, laminar LFPs were measured for 45-75 min until evoked CSD profiles had stabilized (Pre-measurements). The last three recordings before laser stimulation were taken as the referencing pre-measurements. For the C1V1and YFP groups, light fibers were additionally connected to a laser for VTA stimulation. Laser stimulation ( $10 \mathrm{~mW}$ at the optical fiber of the setup) was then coupled and synchronized with each tone presentation for a single measurement $(25 \mathrm{~Hz}, 473 \mathrm{~nm}, 10 \mathrm{~mW} ; 400$ repetitions in total). This measurement was taken as the laser measurement followed by a series of post-measurements ( $>60 \mathrm{~min}$ ). After the experiment, gerbils were perfused with $4 \%$ PFA as described previously ${ }^{85}$ and brains were removed for anatomical and histological validation.

Current source density analysis. Based on the tone-evoked LFPs for each presented stimulus we calculated the second spatial derivative, indicating the laminal evoked current-source density (CSD) distribution in $\mathrm{AI}^{30}$ :

$$
-C S D \approx \frac{\delta^{2} \varnothing(z)}{\delta z^{2}}=\frac{\varnothing(z+n \Delta z)-2 \varnothing(z)+\varnothing(z-n \Delta z)}{(n \Delta z)^{2}}
$$

The formula reflects the relation of the field potential $(\varnothing)$, the spatial coordinate of the cortical laminae $(z)$, the inter-channel distance $(\Delta z, 50 \mu \mathrm{m})$ and the differentiation grid $(n)$. Prior to CSD calculation, LFPs were smoothed using a weighted average of 9 channels (Hamming window, spatial filter kernel size of $400 \mu \mathrm{m}$; linear extrapolation of 3 channels at boundaries $)^{18}$.

CSD profiles revealed patterns of current influx (sinks) and efflux (sources). Based on CSD profiles of the Pre-measurements, cortical layers were assigned to the respective channels of sink components. Early sink components were used as indicators of layers III/IV, $\mathrm{Va}$, and $\mathrm{Vb} / \mathrm{VIa}$, whereas later sink activities were assigned to layers I/II, and VIb (Fig. 2; Additional file 2). 
CSD data was also transformed by rectifying and averaging the waveforms of each single channel $(n)$. This average rectified CSD (AVREC) provides a temporal waveform of the overall local columnar transmembrane current flow ${ }^{31,40}$.

$$
A V R E C=\frac{\sum_{i=1}^{n}\left|C S D_{i}\right|(t)}{n}
$$

We further quantified the residue of the CSD defined as the sum of the non-rectified magnitudes divided by the rectified magnitudes for each channel. Thereby, the ResidualCSD quantifies the balance of the transmembrane charge transfer along the recording $\operatorname{axis}^{86}$ and gives rise to the lateral corticocortical contribution to stimulus related activity ${ }^{18}$.

$$
\text { ResidualCSD }=\frac{\sum_{i=1}^{n} C S D_{i}(t)}{n}
$$

In order to quantify the early $(0-50 \mathrm{~ms})$ and late $(80-300 \mathrm{~ms})$ contributions of the AVREC and ResidualCSD, root mean square (RMS) amplitudes were calculated. Based on the previously described layer indexing, averaged layer-dependent CSDs were calculated, which were used for the semi-automatic sink detection (Additional file 2). Briefly, before detection of significant tone-evoked activity, sources were omitted before calculating the mean of CSD traces within a cortical layer. Then, the 1.5 -fold standard deviation (SD) of the baseline $(-200-0 \mathrm{~ms}$ before tone presentation onset for all channels and stimuli) was used to calculate the intercepts of the smoothed (10 ms gaussian sliding window) mean CSD trace. For early tone-evoked sink activity in cortical layers III/IV, Va, and $\mathrm{Vb} / \mathrm{VIa}$, we used an onset of the first intercept between $0-50 \mathrm{~ms}$ after tone onset. For subsequent tone-evoked sink components in layers I/II and VIb onsets were only taken $>50 \mathrm{~ms}$ after tone-onset. Offsets of each sink were defined as the crossings below the $1.5 \mathrm{SD}$ baseline and for each cortical layer. Peak amplitudes of layer-dependent CSD-traces were determined within time windows of on- and offsets from the average of the corresponding CSD channels. For calculating the RMS value of AVREC and ResidualCSD we used fixed time windows of $0-50 \mathrm{~ms}$ after tone-onset for early and $80-300 \mathrm{~ms}$ for late activity.

Data sorting and analysis. Tuning curves of layer-specific CSD-traces, AVREC, and ResidualCSD were averaged according to the best-frequency of each animal separately to plot an averaged group tuning curve. For illustration, all non-BF frequencies above and below the BF were binned pairwise, resulting into the grouping of near-BFs ( $\pm 1-2$ octaves) and non-BFs ( $\pm 3-4$ octaves) in 5 frequency bins [- non-BF, - near-BF, $\mathrm{BF},+$ near- $\mathrm{BF},+$ non-BF]. For further analysis of BF-, near- and non-BF evoked responses, we observed data from time windows before, during, and after laser stimulation. Evoked responses were normalized within each animal using the corresponding mean values of the last three Pre-measurements before laser-stimulation Fig. 2A.

For statistical comparison, parameters of interest were analyzed on a single-trial level using linear-mixed effect (LME) models (R Studio; R 3.5.1) $)^{34}$ binned across measurements as follows: pre 1-3 (-22.5- -7.5 min), laser (0 min), post $1-3(7.5-22.5 \mathrm{~min})$, post $4-5(30-37.5 \mathrm{~min})$, post $6-7(45-52.5 \mathrm{~min})$. Peak amplitudes of single-trial data were determined within the time windows from on- and offsets revealed on averaged CSD traces. LME models were calculated and fitted using normalized data with the lmerTest-package (3.0-1) and a Wald-chi-square-test was performed using the car-package (3.0-2). Animals were used as "by-subject" random effects, to account for the repeated measurements during and across time points whereas measurements and groups were used as fixed effects to explain physiological data. As significance criterion for effects on single-trial level, the normalized signal needed to (i) exceeded a $\pm 10 \%$ criterion compared to Pre-measurement (based on the SEM of the average jitter before laser stimulation), and (ii) show significant difference from control group data revealed by significant fixed effects of the LME models. For slope estimations and comparison between measurements, we used the R-package Ismeans (version 2.27-62) to calculate a least square linear fit for non-, near- and BF data bins and check significant changes in tuning slope properties between measurements.

Anatomical estimation of fiber position. PFA fixated brains of the C1V1 group $(n=7)$ were cut in $50 \mu \mathrm{m}$ thick slices around the region of the VTA. Every second slice was used for Nissl staining to anatomically evaluate the fiber position, whereas the remaining slices were used for immunostaining to determine the distance from fiber tip to areas of tyrosine hydroxylase (TH) and YFP-co-fluorescence with FIJI ImageJ ( $1.51 \mathrm{~g})$. Pictures were taken with a Leica microscope (Axioscop 2, Leica $\mathrm{GmbH}$ ).

Immunostaining was performed as previously described ${ }^{25}$ with a primary antibody against tyrosine hydroxylase (1:1000 rabbit anti-TH, Milipore) and a secondary Alexa Fluor 546 goat-anti-rabbit (1:400 anti-rabbit, Molecular probes) using an antibody diluent (Zytomed Systems). Position of the fiber was estimated using the recently published gerbil brain atlas for reference ${ }^{84}$.

\section{Data availability}

All relevant data are included in the manuscript and the supplementary information. Used data and codes can be obtained from the corresponding authors upon request.

Received: 5 August 2019; Accepted: 16 December 2019;

Published online: 31 December 2019 


\section{References}

1. Ohl, F. W. Role of cortical neurodynamics for understanding the neural basis of motivated behavior - lessons from auditory category learning. Curr. Opin. Neurobiol. 31, 88-94 (2014).

2. Bromberg-Martin, E. S., Matsumoto, M. \& Hikosaka, O. Dopamine in motivational control: rewarding, aversive, and alerting. Neuron 68, 815-34 (2010).

3. Schultz, W. Neuronal Reward and Decision Signals: From Theories to Data. Physiol. Rev. 95, 853-951 (2015).

4. Bao, S., Chan, V. T. \& Merzenich, M. M. Cortical remodelling induced by activity of ventral tegmental dopamine neurons. Nature 412, 79-83 (2001).

5. Hui, G. K. et al. Conditioned tone control of brain reward behavior produces highly specific representational gain in the primary auditory cortex. Neurobiol. Learn. Mem. 92, 27-34 (2009).

6. Budinger, E., Laszcz, A., Lison, H., Scheich, H. \& Ohl, F. W. Non-sensory cortical and subcortical connections of the primary auditory cortex in Mongolian gerbils: bottom-up and top-down processing of neuronal information via field AI. Brain Res. 1220, 2-32 (2008).

7. Dinopoulos, A. \& Parnavelas, J. G. The development of ventral tegmental area (VTA) projections to the visual cortex of the rat. Neurosci. Lett. 134, 12-6 (1991).

8. Gardner, M. P. H., Schoenbaum, G. \& Gershman, S. J. Rethinking dopamine as generalized prediction error. Proc. R. Soc. B Biol. Sci. 285, 20181645 (2018).

9. Ohl, F. W. \& Scheich, H. Learning-induced plasticity in animal and human auditory cortex. Curr. Opin. Neurobiol. 15, 470-477 (2005).

10. Happel, M. F. K. Dopaminergic impact on local and global cortical circuit processing during learning. Behav. Brain Res. 299, 32-41 (2016).

11. Stark, H. \& Scheich, H. Dopaminergic and serotonergic neurotransmission systems are differentially involved in auditory cortex learning: a long-term microdialysis study of metabolites. J. Neurochem. 68, 691-7 (1997).

12. Schicknick, H. et al. Dopamine modulates memory consolidation of discrimination learning in the auditory cortex. Eur. J. Neurosci. 35, 763-774 (2012).

13. Schicknick, H. et al. Dopaminergic modulation of auditory cortex-dependent memory consolidation through mTOR. Cereb. Cortex 18, 2646-2658 (2008).

14. King, A. J., Teki, S. \& Willmore, B. D. B. B. Recent advances in understanding the auditory cortex. F1000Research 7, 1555 (2018).

15. Campbell, M. J., Lewis, D. A., Foote, S. L. \& Morrison, J. H. Distribution of choline acetyltransferase-, serotonin-, dopamine-?hydroxylase-, tyrosine hydroxylase-immunoreactive fibers in monkey primary auditory cortex. J. Comp. Neurol. 261, 209-220 (1987).

16. Jacob, S. N. \& Nienborg, H. Monoaminergic Neuromodulation of Sensory Processing. Front. Neural Circuits 12, 51 (2018).

17. Happel, M. F. K., Deliano, M., Handschuh, J. \& Ohl, F. W. Dopamine-Modulated Recurrent Corticoefferent Feedback in Primary Sensory Cortex Promotes Detection of Behaviorally Relevant Stimuli. J. Neurosci. 34, 1234-1247 (2014).

18. Happel, M. F. K., Jeschke, M. \& Ohl, F. W. Spectral Integration in Primary Auditory Cortex Attributable to Temporally Precise Convergence of Thalamocortical and Intracortical Input. J. Neurosci. 30, 11114-11127 (2010).

19. Schaefer, M. K., Hechavarría, J. C. \& Kössl, M. Quantification of mid and late evoked sinks in laminar current source density profiles of columns in the primary auditory cortex. Front. Neural Circuits 9, 1-16 (2015).

20. Happel, M. F. K. \& Ohl, F. W. Compensating Level-Dependent Frequency Representation in Auditory Cortex by Synaptic Integration of Corticocortical Input. PLoS ONE 12, e0169461 (2017).

21. Liu, B. H., Wu, G. K., Arbuckle, R., Tao, H. W. \& Zhang, L. I. Defining cortical frequency tuning with recurrent excitatory circuitry. Nat. Neurosci. 10, 1594-1600 (2007).

22. Guo, S. et al. Optogenetic activation of the excitatory neurons expressing CaMKII $\alpha$ in the ventral tegmental area upregulates the locomotor activity of free behaving rats. Biomed Res. Int. 2014, 687469 (2014).

23. Budinger, E. \& Scheich, H. Anatomical connections suitable for the direct processing of neuronal information of different modalities via the rodent primary auditory cortex. Hear. Res. 258, 16-27 (2009).

24. Lippert, M. T. et al. Optogenetic Intracranial Self-Stimulation as a Method to Study the Plasticity-Inducing Effects of Dopamine. Handbook of Behavioral Neuroscience 28, 311-326 (2018).

25. Brocka, M. et al. Contributions of dopaminergic and non-dopaminergic neurons to VTA-stimulation induced neurovascular responses in brain reward circuits. Neuroimage 177, 88-97 (2018).

26. Helbing, C., Brocka, M., Scherf, T., Lippert, M. T. \& Angenstein, F. The role of the mesolimbic dopamine system in the formation of blood-oxygen-level dependent responses in the medial prefrontal/anterior cingulate cortex during high-frequency stimulation of the rat perforant pathway. J. Cereb. Blood Flow Metab. 36, 2177-2193 (2016).

27. Stujenske, J. M., Spellman, T. \& Gordon, J. A. Modeling the Spatiotemporal Dynamics of Light and Heat Propagation for In Vivo Optogenetics. Cell Rep. 12, 525-534 (2015).

28. Yizhar, O., Fenno, L. E., Davidson, T. J., Mogri, M. \& Deisseroth, K. Optogenetics in neural systems. Neuron 71, 9-34 (2011).

29. Arias-Gil, G., Ohl, F. W., Takagaki, K. \& Lippert, M. T. Measurement, modeling, and prediction of temperature rise due to optogenetic brain stimulation. Neurophotonics 3, 045007 (2016).

30. Mitzdorf, U. Current source-density method and application in cat cerebral cortex: investigation of evoked potentials and EEG phenomena. Physiol. Rev. 65, 37-100 (1985).

31. Schroeder, C. E., Metha a, D. \& Givre, S. J. A spatiotemporal profile of visual system activation revealed by current source density analysis in the awake macaque. Cereb. Cortex 8, 575-592 (1998).

32. Szymanski, F. D., Garcia-Lazaro, J. A. \& Schnupp, J. W. H. Current Source Density Profiles of Stimulus-Specific Adaptation in Rat Auditory Cortex. J. Neurophysiol. 102, 1483-1490 (2009).

33. Kroener, S., Chandler, L. J., Phillips, P. E. M. \& Seamans, J. K. Dopamine Modulates Persistent Synaptic Activity and Enhances the Signal-to-Noise Ratio in the Prefrontal Cortex. PLoS ONE 4, e6507 (2009).

34. Chang, A., Bosnyak, D. J. \& Trainor, L. J. Beta oscillatory power modulation reflects the predictability of pitch change. Cortex 106, 248-260 (2018).

35. Owen, S. F., Liu, M. H. \& Kreitzer, A. C. Thermal constraints on in vivo optogenetic manipulations. Nat. Neurosci. 22, $1061-1065$ (2019).

36. Johnson, S. W. \& North, R. A. Two types of neurone in the rat ventral tegmental area and their synaptic inputs. J. Physiol. 450, 455-68 (1992).

37. Paladini, C. A. \& Tepper, J. M. GABAA and GABAB antagonists differentially affect the firing pattern of substantia nigra dopaminergic neurons in vivo. Synapse 32, 165-176 (1999).

38. Tan, K. R. et al. GABA Neurons of the VTA Drive Conditioned Place Aversion. Neuron 73, 1173-1183 (2012).

39. Van Zessen, R., Phillips, J. L., Budygin, E. A. \& Stuber, G. D. Activation of VTA GABA Neurons Disrupts Reward Consumption. Neuron 73, 1184-1194 (2012).

40. Givre, S. J. J., Schroeder, C. E. E. \& Arezzo, J. C. C. Contribution of extrastriate area V4 to the surface-recorded flash VEP in the awake macaque. Vision Res. 34, 415-28 (1994).

41. Intskirveli, I., Joshi, A., Vizcarra-Chacón, B. J. \& Metherate, R. Spectral breadth and laminar distribution of thalamocortical inputs to A1. J. Neurophysiol. 115, 2083-2094 (2016). 
42. Bruno, R. M. \& Sakmann, B. Cortex Is Driven by Weak but Synchronously Active Thalamocortical Synapses. Science (80-.). 312, $1622-1627$ (2006).

43. Adesnik, H. \& Naka, A. Cracking the Function of Layers in the Sensory Cortex. Neuron 100, 1028-1043 (2018).

44. Harris, K. D. \& Mrsic-Flogel, T. D. Cortical connectivity and sensory coding. Nature 503, 51-58 (2013).

45. Castejon, C., Barros-Zulaica, N. \& Nuñez, A. Control of Somatosensory Cortical Processing by Thalamic Posterior Medial Nucleus: A New Role of Thalamus in Cortical Function. PLoS ONE 11, e0148169 (2016).

46. Jarvis, S., Nikolic, K. \& Schultz, S. R. Neuronal gain modulability is determined by dendritic morphology: A computational optogenetic study. PLOS Comput. Biol. 14, e1006027 (2018).

47. Sánchez-González, M. A., García-Cabezas, M. A., Rico, B. \& Cavada, C. The primate thalamus is a key target for brain dopamine. J. Neurosci. 25, 6076-83 (2005).

48. Varela, C. Thalamic neuromodulation and its implications for executive networks. Front. Neural Circuits 8, 69 (2014).

49. Deliano, M. et al. Dopaminergic neuromodulation of high gamma stimulus phase-locking in gerbil primary auditory cortex mediated by D1/D5-receptors. Eur. J. Neurosci. https://doi.org/10.1111/ejn.13898 (2018).

50. Sugimoto, S., Sakurada, M., Horikawa, J. \& Taniguchi, I. The columnar and layer-specific response properties of neurons in the primary auditory cortex of Mongolian gerbils. Hear. Res. 112, 175-185 (1997).

51. De Martino, F. et al. Frequency preference and attention effects across cortical depths in the human primary auditory cortex. Proc. Natl. Acad. Sci. USA 112, 16036-41 (2015).

52. Constantinople, C. M. \& Bruno, R. M. Deep Cortical Layers Are Activated Directly by Thalamus. Science (80-.). 340, 1591-1594 (2013).

53. Zhao, W. J., Kremkow, J. \& Poulet, J. F. A. Translaminar Cortical Membrane Potential Synchrony in Behaving Mice. Cell Rep. https:// doi.org/10.1016/j.celrep.2016.05.026 (2016)

54. Pais-Vieira, M., Chiuffa, G., Lebedev, M., Yadav, A. \& Nicolelis, M. A. L. Building an organic computing device with multiple interconnected brains. Sci. Rep. 5, 11869 (2015).

55. Biane, J. S., Takashima, Y., Scanziani, M., Conner, J. M. \& Tuszynski, M. H. Thalamocortical Projections onto Behaviorally Relevant Neurons Exhibit Plasticity during Adult Motor Learning. Neuron 89, 1173-1179 (2016).

56. Froemke, R. C., Merzenich, M. M. \& Schreiner, C. E. A synaptic memory trace for cortical receptive field plasticity. Nature $\mathbf{4 5 0}$, $425-429(2007)$

57. Liu, C. L., Gao, M., Jin, G. Z. \& Zhen, X. GABA Neurons in the Ventral Tegmental Area Responding to Peripheral Sensory Input. PLoS ONE 7, 1-8 (2012).

58. Nair-Roberts, R. G. et al. Stereological estimates of dopaminergic, GABAergic and glutamatergic neurons in the ventral tegmental area, substantia nigra and retrorubral field in the rat. Neuroscience 152, 1024-31 (2008).

59. Sesack, S. R. \& Grace, A. A. Cortico-Basal Ganglia reward network: microcircuitry. Neuropsychopharmacology 35, 27-47 (2010).

60. Wang, H.-L., Qi, J., Zhang, S., Wang, H. \& Morales, M. Rewarding Effects of Optical Stimulation of Ventral Tegmental Area Glutamatergic Neurons. J. Neurosci. https://doi.org/10.1523/JNEUROSCI.3428-15.2015 (2015).

61. Gorelova, N., Mulholland, P. J., Chandler, L. J. \& Seamans, J. K. The glutamatergic component of the mesocortical pathway emanating from different subregions of the ventral midbrain. Cereb. Cortex 22, 327-36 (2012).

62. Yoo, J. H. et al. Ventral tegmental area glutamate neurons co-release GABA and promote positive reinforcement. Nat. Commun. 7, 1-13 (2016).

63. Stuber, G. D., Hnasko, T. S., Britt, J. P., Edwards, R. H. \& Bonci, A. Dopaminergic Terminals in the Nucleus Accumbens But Not the Dorsal Striatum Corelease Glutamate. J. Neurosci. 30, 8229-8233 (2010).

64. El Mestikawy, S., Wallén-Mackenzie, A., Fortin, G. M., Descarries, L. \& Trudeau, L.-E. From glutamate co-release to vesicular synergy: vesicular glutamate transporters. Nat. Rev. Neurosci. 12, 204-216 (2011).

65. Hnasko, T. S., Hjelmstad, G. O., Fields, H. L. \& Edwards, R. H. Ventral Tegmental Area Glutamate Neurons: Electrophysiological Properties and Projections. J. Neurosci. 32, 15076-15085 (2012).

66. Lapish, C. C., Seamans, J. K. \& Judson Chandler, L. Glutamate-Dopamine Cotransmission and Reward Processing in Addiction. Alcohol. Clin. Exp. Res. 30, 1451-1465 (2006).

67. Lavin, A. et al. Mesocortical dopamine neurons operate in distinct temporal domains using multimodal signaling. J. Neurosci. 25, 5013-23 (2005).

68. Mylius, J. et al. Fast transmission from the dopaminergic ventral midbrain to the sensory cortex of awake primates. Brain Struct. Funct, https://doi.org/10.1007/s00429-014-0855-0 (2015).

69. Seamans, J. K. \& Yang, C. R. The principal features and mechanisms of dopamine modulation in the prefrontal cortex. Prog. Neurobiol. 74, 1-58 (2004).

70. Phillipson, O. T., Kilpatrick, I. C. \& Jones, M. W. Dopaminergic innervation of the primary visual cortex in the rat, and some correlations with human cortex. Brain Res. Bull. 18, 621-633 (1987).

71. Lewis, D. A., Campbell, M. J., Foote, S. L., Goldstein, M. \& Morrison, J. H. The Distribution of Tyrosine Hydroxylase-lmmunoreactive Fibers in Primate Neocortex Is Widespread but Regionally Specific. J. Neurosci. 7, 279-290 (1987).

72. Campbell, A. D., Kohl, R. R. \& McBride, W. J. Serotonin-3 receptor and ethanol-stimulated somatodendritic dopamine release. Alcohol. https://doi.org/10.1016/S0741-8329(96)00069-9 (1996).

73. Lidow, M. S., Goldman-Rakic, P. S., Gallager, D. W. \& Rakic, P. Distribution of dopaminergic receptors in the primate cerebral cortex: Quantitative autoradiographic analysis using [3H]raclopride, [3H]spiperone and [3H]SCH23390. Neuroscience 40, 657-671 (1991).

74. Williams, S. M. \& Goldman-Rakic, P. S. Characterization of the dopaminergic innervation of the primate frontal cortex using a dopamine-specific antibody. Cereb. Cortex. https://doi.org/10.1093/cercor/3.3.199 (1993)

75. Blundon, J. A. \& Zakharenko, S. S. Presynaptic Gating of Postsynaptic Synaptic Plasticity at thalamocortical synapses: a plasticity filter in the adult auditory cortex. Neurosci. 19, 465-478 (2013).

76. Izhikevich, E. M. Solving the distal reward problem through linkage of STDP and dopamine signaling. Cereb. Cortex. https://doi. org/10.1093/cercor/bhl152 (2007).

77. Mitlöhner, J. et al. Dopamine modulates the integrity of the perisynaptic extracellular matrix at excitatory synapses. bioRxiv 722454 . https://doi.org/10.1101/722454 (2019).

78. Polley, D. B., Steinberg, E. E. \& Merzenich, M. M. Perceptual learning directs auditory cortical map reorganization through topdown influences. J. Neurosci. 26, 4970-82 (2006).

79. Caras, M. L. \& Sanes, D. H. Top-down modulation of sensory cortex gates perceptual learning. Proc. Natl. Acad. Sci. 114, 9972-9977 (2017).

80. Froemke, R. C. et al. Long-term modification of cortical synapses improves sensory perception. Nat. Neurosci. 16, 79-88 (2013)

81. Sohoglu, E. \& Chait, M. Detecting and representing predictable structure during auditory scene analysis. Elife 5, (2016).

82. Kato, H. K., Gillet, S. N. \& Isaacson, J. S. Flexible Sensory Representations in Auditory Cortex Driven by Behavioral Relevance. Neuron 88, 1027-1039 (2015).

83. Hull, C. L. Principles of Behavior: An Introduction to Behavior Theory. Appleton-Century-Crofts, Inc. https://doi.org/10.1037/ h0051597 (1944)

84. Radtke-Schuller, S. et al. Brain atlas of the Mongolian gerbil (Meriones unguiculatus) in CT/MRI-aided stereotaxic coordinates. Brain Struct. Funct. 221, 1-272 (2016). 
85. Saldeitis, K., Happel, M. F. K., Ohl, F. W., Scheich, H. \& Budinger, E. Anatomy of the auditory thalamocortical system in the mongolian gerbil: Nuclear origins and cortical field-, layer-, and frequency-specificities. J. Comp. Neurol. 522, 2397-2430 (2014).

86. Harding, G. W. The currents that flow in the somatosensory cortex during the direct cortical response. Exp. Brain Res. 90, 29-39 (1992).

\section{Acknowledgements}

We would like to thank Janet Stallmann, Anja Gürke and Kathrin Ohl for assistance with surgeries, histology-and immunostaining of brain slices. We would like to thank PD Dr. Eike Budinger and Dr. Julia Henschke for their help and assistance in anatomical questions. Many thanks to our interns for their assistance with anatomical reconstructions: Tarik Drewes, Katharina Eick, Vincent Koop, Jan-Niklas Löbner, Julia Rieger and Ceylan Steinecke. This project and corresponding research have been funded by the Leibniz Association WGL by the Leibniz Postdoctoral Network, LPN (to MTL and MFKH) and, the Deutsche Forschungsgemeinschaft DFG by the SFB779 (to MFKH and FWO) and SPP1665 (to MTL and FWO).

\section{Author contributions}

F.W.O., M.T.L. and M.F.K.H. designed research. M.G.K.B. and K.E.D. performed electrophysiology. M.G.K.B. and S.V. performed histology and behavioral experiments. M.G.K.B., K.E.D., M.K., M.D. and M.F.K.H. analyzed data. MGKB, K.E.D. and M.D. contributed analytic tools. M.G.K.B. and M.F.K.H. prepared the figures and wrote the initial draft. M.G.K.B., K.E.D., F.W.O., M.T.L. and M.F.K.H. wrote the manuscript. All authors reviewed and revised the manuscript.

\section{Competing interests}

The authors declare no competing interests.

\section{Additional information}

Supplementary information is available for this paper at https://doi.org/10.1038/s41598-019-56926-6.

Correspondence and requests for materials should be addressed to M.G.K.B. or M.F.K.H.

Reprints and permissions information is available at www.nature.com/reprints.

Publisher's note Springer Nature remains neutral with regard to jurisdictional claims in published maps and institutional affiliations.

(c) (i) Open Access This article is licensed under a Creative Commons Attribution 4.0 International License, which permits use, sharing, adaptation, distribution and reproduction in any medium or format, as long as you give appropriate credit to the original author(s) and the source, provide a link to the Creative Commons license, and indicate if changes were made. The images or other third party material in this article are included in the article's Creative Commons license, unless indicated otherwise in a credit line to the material. If material is not included in the article's Creative Commons license and your intended use is not permitted by statutory regulation or exceeds the permitted use, you will need to obtain permission directly from the copyright holder. To view a copy of this license, visit http://creativecommons.org/licenses/by/4.0/.

(C) The Author(s) 2019 